\title{
ULTRASONIC INVESTIGATION OF PLASTICALLY DEFORMED STEEL-CONTRIBUTION TO ANALYSIS OF DEGRADATION INFLUENCE
}

Study of degradation influence on material properties of some steel components is very important because of their serviceable life. One of the most important side of the degradation process considering operation conditions is cumulated plastic deformation. The ultrasonic investigation of materials through the ultrasound attenuation and velocity measurements have been proved an effective tool of the study of structural changes caused also by plastic deformation due to the degradation process. In the contribution we present the results of both the frequency dependence of ultrasound attenuation and velocity measurements of steel samples (38ChN3MFA) with different relative plastic deformation (0,1.5, 3 and $6 \%)$. The results are compared also with the measurements obtained by some other techniques, including the measurement of electrical conductivity, and the rate of positron-electron annihilation and breakage toughness.

\section{Introduction}

Quantities characterising the propagation of high-frequency acoustic waves (ultrasound) in solid materials can be determined from the investigation of dependence of both acoustic waves velocity and attenuation on individual variables describing investigated properties of existing materials. The relation of velocity and attenuation of ultrasonic waves to various properties referred to intrinsic structure of solid material can then enable their study by means of ultrasonic velocity and attenuation measurement. Among these properties befit mainly elastic properties of materials, which reflect directly their intrinsic structure $[1,2]$.

Using the measurement of acoustic waves velocities and known values of density the elastic constants of solid materials, which are connected directly with inter-atomic forces, can be determined. The acoustic waves attenuation very sensitively reflects material intrinsic structure, not only the type of structure but also the changes due to defects occurrence (dislocations, grains etc.) Acoustic attenuation in metal materials depends on the way of preparation and further thermal treatment, that can influence a grain magnitude as well as the generation of dislocations or other defects. The attenuation also reflects permanent changes in material arising due to its strain [3].

In the case of material with a grain structure, dislocations or other defects the attenuation is caused by the dispersion, the frequency dependence of which depends on the rate of wavelength and mean defect size [1].

Presented contribution is orientated on analysis of degradation influence of material properties (steel 38ChN3MFA) with respect

\footnotetext{
* ${ }^{1}$ P. Bury, ${ }^{2} \check{S}$. Barta, ${ }^{3}$ V. Magula, ${ }^{2}$ V. Slugen, ${ }^{3}$ T. Šmida

${ }^{1}$ Department of Physics, Faculty of Electrical Engineering, Žilina University, 01026 Žilina

${ }^{2}$ Department of Physics, Faculty of Electroengineering and Informatics, Slovak Technical University, 81219 Bratislava

${ }^{3}$ Welding Research Institute, Račianska 71, 83259 Bratislava
}

to serviceable life of produced components (for example girder M140) by investigation of cumulated plastic deformation. The structure changes of existing material were investigated for various degrees of plastic deformation using both attenuation frequency dependence and ultrasound velocity measurements. Obtained results were in detail compared with results obtained by other methods on the same samples.

\section{Experiment}

Investigated material was chosen from non-operated girder M140 and four sample series corresponding to four different material conditions were prepared; original $(0 \%$ of relative plastic deformation in strain); $1.5 \%, 3 \%$ and $6 \%$ of tensile deformation Nevertheless, the $6 \%$ relative plastic deformation corresponded to the strain just before the tensile strength level [4].

Samples for the acoustical investigation were prepared in the cylinder shape with diameter $d=13 \mathrm{~mm}$ and high $h=8.65 \mathrm{~mm}$. The velocity and attenuation were measured for ultrasonic wave propagated in the direction of cylinder axis (x) and there by in deformation direction, but also in the direction perpendicular to cylinder axis(y) with faces separated by $l=10.78 \mathrm{~mm}$.

Acoustic measurements of the both velocity and attenuation were made in frequency range of $10-100 \mathrm{MHz}$ using pulse method and longitudinal acoustic waves generated by quartz transducers. All measurements were performed at room temperature. 


\section{Results and discussion}

The results of velocity measurement for both propagating directions and corresponding elastic modules for the cylinder axis direction are summarised in Table 1 . The velocity measurements were made at frequency of $12 \mathrm{MHz}$ with accuracy better than $2 \cdot 10^{-3}$. The graph of frequency dependence of ultrasonic attenuation measured for individual samples is in Fig. 1 .

Ultrasonic velocity measurements for all samples and two propagating directions ( $x$ and $y$ ) and elastic modules for propagating direction $x$.

\begin{tabular}{|c|c|c|c|}
\hline plast. defor. (\%) & $v_{L(X)}[\mathrm{m} / \mathrm{s}]$ & $v_{L(Y)}[\mathrm{m} / \mathrm{s}]$ & $E_{x}[\mathrm{~Pa}]$ \\
\hline 0 & 5553 & 5580 & $24.14 \times 10^{10}$ \\
\hline 1.5 & 5553 & 5580 & $24.14 \times 10^{10}$ \\
\hline 3 & 5537 & 5595 & $24.00 \times 10^{10}$ \\
\hline 6 & 5676 & 5603 & $25.22 \times 10^{10}$ \\
\hline
\end{tabular}

The results obtained by the measurements of attenuation and velocity correlate considerably with results of other measurements obtained on the same samples, mainly the measurement of positronelectron annihilation rate, measurement of impact toughness by measuring of transient temperature and measurement of static breakage toughness. The most important results of mentioned measurements are summarized in Table 2 .

Summarized results of measurement of plastic deformation influence on some physical characteristics. Here, $750 \%$ and $T 60 J$ are the transient temperatures measured at impact work $60 \mathrm{~J}$ and temperature of $50 \%$ plastic breakage, respectively, $K_{0,2}$ is the static breakage toughness for crack growth $0.2 \mathrm{~mm}, \rho$ is the electric resistivity and $\tau$ is the positron lifetime.

\begin{tabular}{|c|c|c|c|c|}
\hline $\begin{array}{c}\text { Measured } \\
\text { characteristic }\end{array}$ & \multicolumn{4}{|c|}{ Plastic deformation [\%] } \\
\cline { 2 - 5 }$T 50 \%\left[{ }^{\circ} \mathrm{C}\right]$ & -82 & -74 & -72 & -65 \\
\hline $\operatorname{T60J}\left[{ }^{\circ} \mathrm{C}\right]$ & -63 & -68 & -53 & -42 \\
\hline$K_{0,2}[\mathrm{MPa} \sqrt{ }]$ & 182 & 190 & 159 & 152 \\
\hline $10^{8} \rho[\Omega \mu]$ & 29.4 & 29.5 & 29.1 & 28.7 \\
\hline$\tau[\mathrm{ps}]$ & 174.5 & 172.5 & 179.7 & 178.3 \\
\hline
\end{tabular}

On the base of results of impact and static breakage toughness tests, it is necessary to state that a plastic deformation of girder material in the range of $0-6 \%$ shows only lightly expressively on its resistance against a brittle damage. In spite of a relatively small differences in measured values and with consideration of results possible dispersion, the expected slow increase of transient temperature and slow decrease of breakage toughness values due to the plastic deformation up to $6 \%$ was indeed confirmed, but from the respect to safety operation of girders it is not important.

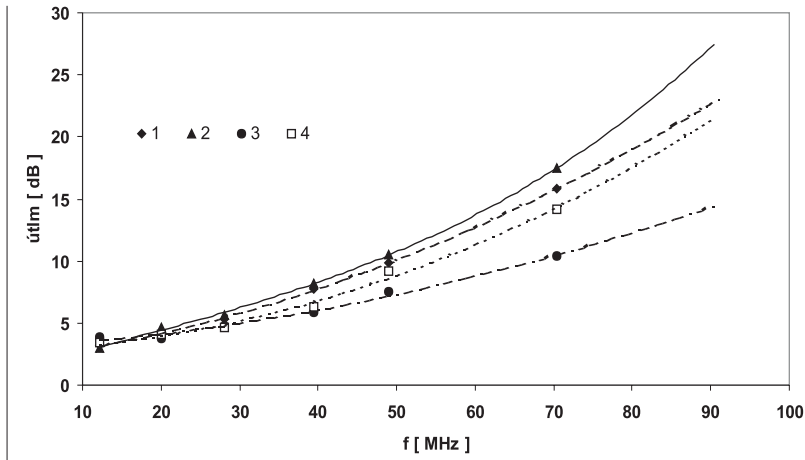

Fig. 1. Frequency dependence of ultrasonic attenuation for the samples with different value of relative plastic deformation: $0 \%(4) ; 1.5 \%(1) ; 3 \%(2)$ and $6 \%$ (3).

The test of static breakage toughness that presents at $20{ }^{\circ} \mathrm{C}$ more reproducible results simultaneously indicated that the marked properties degradation occurs between 1.5 and $3.0 \%$ of plastic deformation. The differences between the values of static breakage toughness of original material and material after $1.5 \%$ deformation, as well as between materials after $3 \%$ and $6 \%$ deformation, respectively, were intro-dispersion of measurements.

The relative small influence of plastic deformation in the range of $0-6 \%$ is with respect to mechanical properties of the girder [4] understanding. It could he seen from the tensile pattern that there is not any deformation hardening in the range of $0-1.5 \%$. In addition the $1.5 \%$ relative tensile deformation represents only small fraction of the material deformation efficiency (tensibility about $18 \%$ and contraction almost $60 \%$ ). Therefore there is no reason for its important influence on the toughness.

The decrease of the toughness after plastic deformation in the range of $1.5-3 \%$ is with largest probability caused by the strength matrices development due to the relative important deformation hardening. It is evident that the deformation hardening is the most marked at deformations in the range of $1.5-3 \%$. From the volume constancy of plastic deformation but follows, that the contraction value at $3 \%$ relative tensile deformation range only on the level below $5 \%$ of total material plasticity. So small loss of plasticity due to the considering natural measurements dispersion could be hardly registered on the value of static breakage toughness and transient temperature, respectively.

On the other side, the rate of deformation hardening over $\sim 3 \%$ of relative tensile deformation again decreases, which indicates a relatively small increase of strength matrices behind the strength limit. With respect to small increase of strength matrices, the influence of cumulated plastic deformation would be registered more significantly only in the case of considerably higher values of plastic deformation.

The results obtained by measurements of both frequency dependence of ultrasonic signal and positron-electron annihilation exhibited already at preliminary measurements to be promising. 
The values of both physical characteristics in the range of $0-1.5 \%$ of relative tensile deformation were practically constant. Measured differences are in the frame of measurement error, which correlates with zero matrices hardening in the initial stages of plastic deformation.

In the range of $1.5-3 \%$ of plastic deformation the marked increase of ultrasonic attenuation over $50 \mathrm{MHz}$ was registered similarly as increase of mean positron lifetime, which indicates a change in the state of lattice defects. Taking into consideration the fact, that the total plastic deformation depends directly on the density of dislocation lines in matrices, that should be necessarily changed during initial deformation phases the behaviour of both parameters are with largest probability the mark of raising sensitivity of both methods on the cluster creation of intersected dislocations (dislocation forest) during initial stages of plastic deformation.

However, the attenuation values measured in the range of $3-6 \%$ indicate comparing with the values of mean positron lifetime different sensitivity on defects number and configuration - the mean positron lifetime is practically constant while the attenuation significantly decreases. The small deformation hardening recognised in existing range indicates probably the beginning of creation of that areas with reduced density of dislocation lines and these areas alternate with areas with increasing dislocation density. It is possible that the method at ultrasonic attenuation measurement is more sensitive to the creation at dislocation substructure, however this assumption should be verified by additional measurements.

\section{Conclusion}

In conclusion we can state, that methods of ultrasonic attenuation and velocity measurement definitely recorded the region of $1.5-3 \%$ plastic deformation, which is not indication of important reduction of material toughness, but it indicates the formation of degradation proces, that could manifest negatively at higher deformation values and the speed of which could be much higher at cumulated values of $3-6 \%$ with respect to the small speed of deformation hardening. From the point of view of further operation of girder M 140 it was found that a plastic deformation does not cause important change of breakage properties and is not manifested on equipment safety. Regarding to possible utilisation of this method for monitoring of cumulated plastic deformation as well as also girders safety (residual lifetime), we can state that the measurement of ultrasonic attenuation over frequency $50 \mathrm{MHz}$ after results verification using larger sample set could probably serve as check non-destructive method for monitoring of the rise of cumulated plastic deformation that does not influence indeed the girder residual lifetime but indicates at the values $\sim 3 \%$ the beginning of the stage when the value of cumulated plastic deformation can increase beyond control at small increase of nominal loading.

\section{References:}

[1] MASON, W. P.: Physical Acoustics, Vol. IV, Academic Press, 1966

[2] PAPADAKIS, E. P.: Ultrasonic Instruments and Devices, Academic Press, 2001

[3] TRUEL, R., ELBAUM, CH., CHICK, B. B.: Ultrasonic Methods in Solid State Physics, Academic Press, 1969

[4] HAMÁK, I., et. al.: Residual lifetime of girders M 140 after long-time operation, Order No. 222/2167 VÚZ Bratislava, September 2000 